\title{
Advances in 3DTV: Theory and Practice
}

\author{
Xenophon Zabulis, ${ }^{1}$ Irene Cheng, ${ }^{2}$ Nikolaos Grammalidis, ${ }^{3}$ Georgios A. Triantafyllidis, ${ }^{3}$ \\ and Pietro Zanuttigh ${ }^{4}$
}

${ }^{1}$ ICS-FORTH, Greece

${ }^{2}$ University of Alberta, Canada

${ }^{3}$ ITI-CERTH, Greece

${ }^{4}$ University of Padova, Italy

Correspondence should be addressed to Xenophon Zabulis, zabulis@ics.forth.gr

Received 21 March 2010; Accepted 21 March 2010

Copyright (C) 2010 Xenophon Zabulis et al. This is an open access article distributed under the Creative Commons Attribution License, which permits unrestricted use, distribution, and reproduction in any medium, provided the original work is properly cited.

Being a goal over decades, the extension of television, and visual communications in general, to the third dimension (3DTV) has been almost reached. Currently, 3D motion pictures are shown in theatres and the first 3D broadcasts are being planned to initiate in the next few years. Albeit the progress, state of the art has not yet reached the goal of acquiring a three-dimensional scene in full detail and creating a precise optical duplicate at remote site in real-time. Limitations in reconstruction accuracy and visual quality as well as user acceptance of pertinent technologies have, to date, prevented the creation of infrastructures for the delivery of 3D content to mass markets. Thereby, relevant scientific research is at the center of interest regarding the improvement of current 3DTV technologies.

The goal of 3DTV involves the collaboration of researchers in multiple scientific disciplines as well as the integration of pertinent results, in order to provide end-users with a fully functional system. The tasks of "capture and representation of 3D scene information," "representation of the digital 3DTV signal," "storage and transmission of the digital 3DTV signal," as well as "display of the reproduced 3D scene" constitute a chain of operations that are essential for reaching this goal. In this context, this special issue presents contributions along the whole chain of computational tasks that are required to implement 3DTV. In addition, the last two papers provide with contributions that go beyond the simple presentation of $3 \mathrm{D}$ visual data and propose ways of interacting with them.
The paper "Multi-view shooting geometry for multiscopic rendering with controlled distortion" introduces a complete analysis of the geometrical quality of $3 \mathrm{D}$ content. The article compares the perceived depth with scene depth obtained from the viewing and shooting (acquisition) geometries, yielding a depth distortion model whose parameters are expressed by the geometrical characteristics of shooting and rendering devices. These expressions are then inverted, in order to design convenient shooting layouts yielding chosen distortions on specific rendering devices.

The paper "Near real time estimation of super-resolved depth and all-in-focus images from a plenoptic camera using graphic processing units (GPU)," provides a near real-time method for the employment of plenoptic video-cameras in the acquisition of all-in-focus color images with accompanying depth values. The proposed method overcomes low-resolution deficiencies of previous methods, while its output can be directly provided as input to autostereoscopic displays.

Occlusions are a characteristic problem in disparity estimation, in binocular and multiview stereo. The paper "An occlusion approach with consistency constraint for multiscopic depth extraction" addresses this issue, handling occlusions for improved disparity estimation from multiple images acquired by a parallel camera geometry. The proposed technique ensures the consistency of disparity maps and its application demonstrated on 
both a correlation-based and a graph-cut based stereo approach.

The need for fast and compact representations for 3D animations raises two issues: (1) how to classify portions of an object into moving and stationary and (2) how to represent nonrigid motion for moving portions. In this context, the paper "A compact representation for 3D animation using octrees and affine transformations" deals with a new and compact 3D representation for nonrigid objects using motion vectors between consecutive frames. The proposed method employs an affine-based motion representation that uses a rigid octree structure for partitioning.

Multiple Description Coding is one of the most promising approaches for the transmission of video signals over unreliable networks. The paper "A scalable multiple description scheme for $3 \mathrm{D}$ video coding based on the inter layer prediction structure" presents a coding scheme that is based on H.264/SVC that enables the application Multiple Description Coding to 3D video. The proposed method is applicable on both stereo sequences and depth-image-based rendering schemes.

The application of digital holography as a viable solution to $3 \mathrm{D}$ capture and display technology is examined in the paper "Digital holographic capture and opto-electronic reconstruction for 3D displays." The proposed work addresses major challenges in digital holography including (1) the removal of the DC and conjugate image terms, which are features of the holographic recording process, (2) the reduction of speckle noise, a characteristic of a coherent imaging process, (3) increasing the spatial resolution of digital holograms, and (4) replaying captured and/or processed digital holograms using spatial light modulators.

The transmission of 3D scenes over unreliable networks is a challenging topic in digital broadcasting research. In order to maximize the rendered quality, the article entitled "Transmission of 3D scenes over lossy channels" introduces a novel Unequal Error Protection scheme to transmit texture and depth information. Various scene elements are assigned different predefined amounts of redundancy based on their visual impacts. Experimental results demonstrate the effectiveness of the proposed scheme.

A complete multicamera real-time 3D modeling system, Grimage, is introduced in "Multi-camera real-time 3D modeling for telepresence and remote collaboration," which is employed in the creation of new immersive and interactive environments. The system creates in real-time a textured 3D model of the observed scene and utilized this information to compute collisions and reaction forces with virtual objects, enforcing the mechanical presence of the user in the virtual world.

The paper "Stereo 3D mouse cursor: A method for interaction with $3 \mathrm{D}$ objects in a stereoscopic virtual $3 \mathrm{D}$ space" presents a novel approach that applies stereoscopic principles to the classical mouse cursor in order to build a 3D pointing device that allows to effectively navigate within $3 \mathrm{D}$ scenes. The outcome is a novel way to interact with the reconstructed $3 \mathrm{D}$ that matches the requirements of a fully immersive experience.

With research and development on 3D imaging and visual reproduction systems being more active than ever in the past, pertinent technologies are expected to be increasingly employed in the broader context of communication of $3 \mathrm{D}$ visual information.

\author{
Xenophon Zabulis \\ Irene Cheng \\ Nikolaos Grammalidis \\ Georgios A. Triantafyllidis \\ Pietro Zanuttigh
}



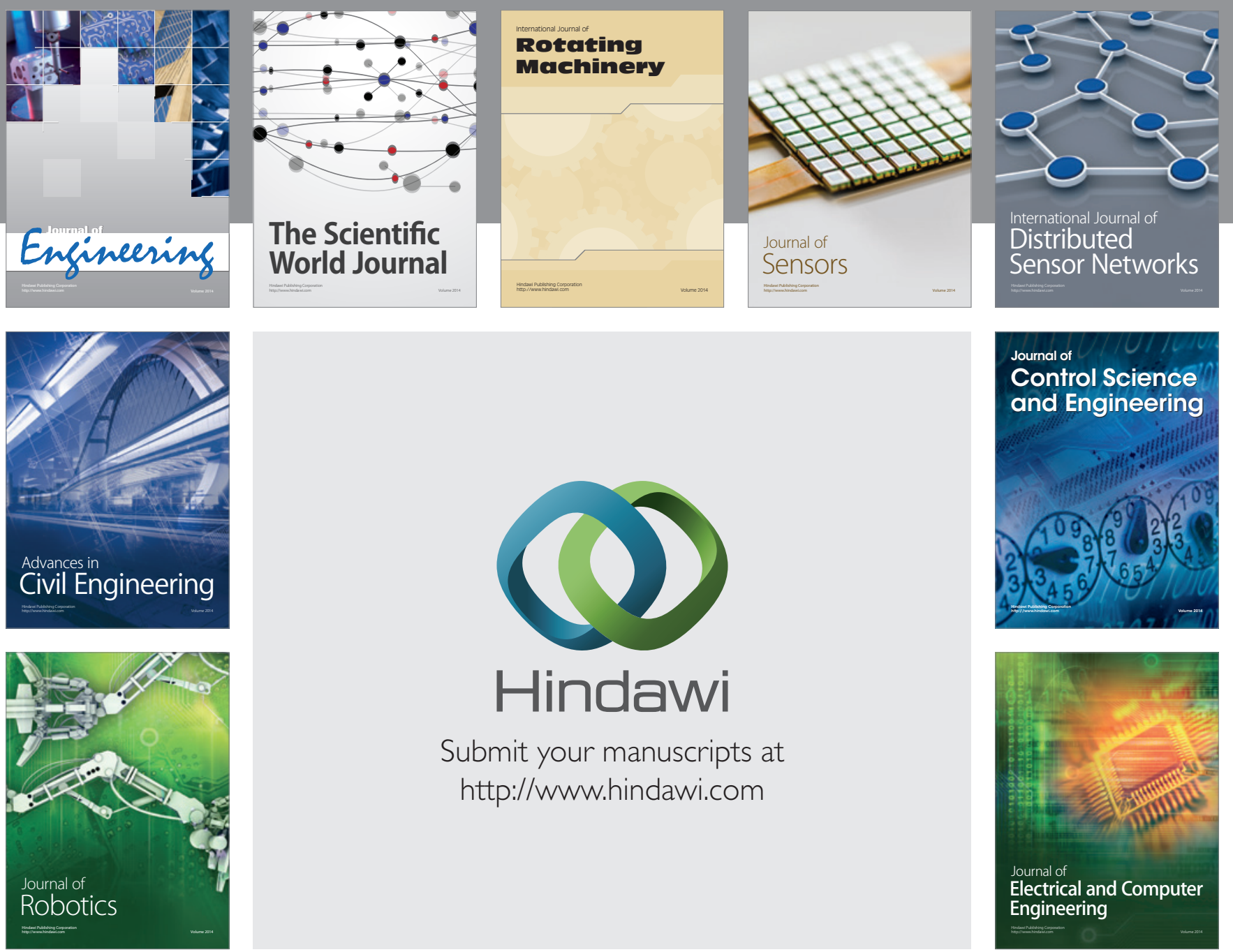

Submit your manuscripts at

http://www.hindawi.com
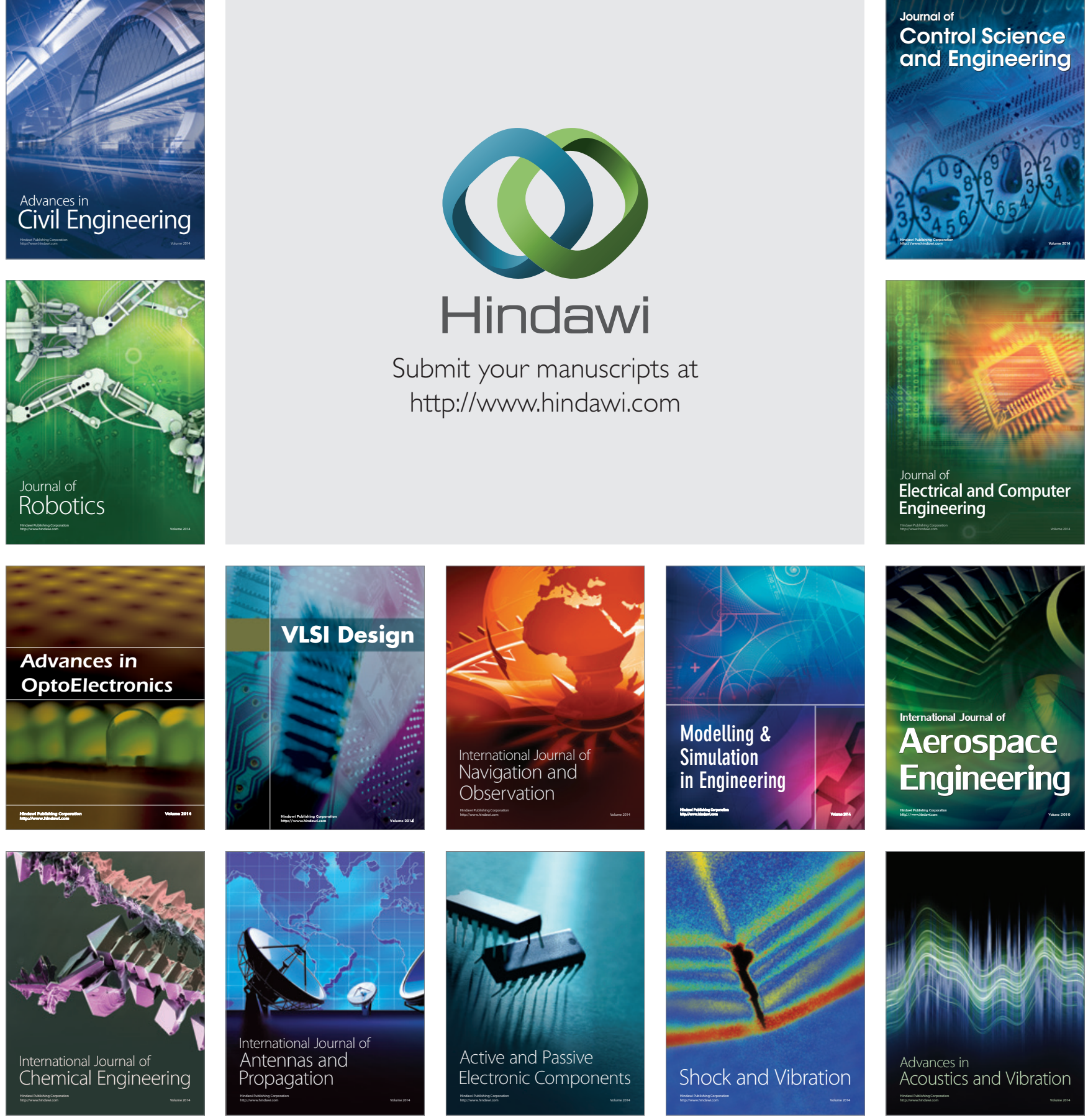